\title{
SOME LOGARITHMICALLY COMPLETELY MONOTONIC FUNCTIONS RELATED TO THE GAMMA FUNCTION
}

\author{
FEng Qi AND BAI-Ni GuO
}

\begin{abstract}
In this article, the logarithmically complete monotonicity of some functions such as $\frac{1}{[\Gamma(x+1)]^{1 / x}}, \quad \frac{[\Gamma(x+1)]^{1 / x}}{x^{\alpha}}, \quad \frac{[\Gamma(x+1)]^{1 / x}}{(x+1)^{\alpha}} \quad$ and $\quad \frac{[\Gamma(x+\alpha+1)]^{1 /(x+\alpha)}}{[\Gamma(x+1)]^{1 / x}}$ for $\alpha \in \mathbb{R}$ on $(-1, \infty)$ or $(0, \infty)$ are obtained, some known results are recovered, extended and generalized. Moreover, some basic properties of the logarithmically completely monotonic functions are established.
\end{abstract}

\section{Introduction}

Recall [23, Chapter XIII] and [41, Chapter IV] that a function $f$ is said to be completely monotonic on an interval $I$ if $f$ has derivatives of all orders on $I$ and

$$
(-1)^{k} f^{(k)}(x) \geq 0
$$

for all $k \geq 0$ on $I$.

For our own convenience, let $\mathcal{C}[I]$ denote the set of completely monotonic functions on $I$.

The well-known Bernstein's Theorem in [41, p. 160, Theorem 12a] states that a function $f$ on $[0, \infty)$ is completely monotonic if and only if there exists a bounded and non-decreasing function $\alpha(t)$ such that

$$
f(x)=\int_{0}^{\infty} e^{-x t} \mathrm{~d} \alpha(t)
$$

converges for $x \in[0, \infty)$. This tells us that $f \in \mathcal{C}[[0, \infty)]$ if and only if it is a Laplace transform of the measure $\alpha$.

Recall also $[5,27,28]$ that a positive function $f$ is said to be logarithmically completely monotonic on an interval $I$ if $f$ has derivatives of all orders on $I$

Received March 30, 2009; Revised June 9, 2009.

2000 Mathematics Subject Classification. Primary 26A48, 33B15; Secondary 26A51, $65 \mathrm{R} 10$.

Key words and phrases. logarithmically completely monotonic function, completely monotonic function, gamma function, basic property. 
and

$$
(-1)^{n}[\ln f(x)]^{(n)} \geq 0
$$

for all $x \in I$ and $n \in \mathbb{N}$.

For simplicity, let $\mathcal{L}[I]$ stand for the set of logarithmically completely monotonic functions on $I$.

Among other things, it is proved in $[6,9,27]$ that a logarithmically completely monotonic function is always completely monotonic, that is, $\mathcal{L}[I] \subset \mathcal{C}[I]$, but not conversely, since a convex function may not be logarithmically convex (see [24, p. 7, Remark. 1.16]).

Recall [41] that a function $f$ defined on $(0, \infty)$ is called a Stieltjes transform if it can be of the form

$$
f(x)=a+\int_{0}^{\infty} \frac{1}{s+x} \mathrm{~d} \mu(s),
$$

where $a$ is a nonnegative number and $\mu$ a nonnegative measure on $[0, \infty)$ satisfying

$$
\int_{0}^{\infty} \frac{1}{1+s} \mathrm{~d} \mu(s)<\infty
$$

The set of Stieltjes transforms is denoted by $\mathcal{S}$.

Motivated by the papers $[28,32]$, it is revealed in [6] that

$$
\mathcal{S} \backslash\{0\} \subset \mathcal{L}[(0, \infty)] \subset \mathcal{C}[(0, \infty)] .
$$

In [6, Theorem 1.1] and [15] it is pointed out that logarithmically completely monotonic functions on $(0, \infty)$ can be characterized as the infinitely divisible completely monotonic functions studied by Horn in [16, Theorem 4.4].

The functions in $\mathcal{L}[I]$ are also characterized by $-\frac{f^{\prime}}{f} \in \mathcal{C}[I]$.

Recently it is found that a finer inclusion

$$
\mathcal{S} \subset \mathcal{C}^{*}[(0, \infty)] \subset \mathcal{L}[(0, \infty)] \subset \mathcal{C}[(0, \infty)]
$$

had been established in [7, Section 14.2 , pp. 122-127] and [37], where $\mathcal{C}^{*}[(0, \infty)]$ denotes the set

$$
\left\{f(x) \mid\left[\frac{1}{f(x)}\right]^{\prime} \in \mathcal{C}[(0, \infty)]\right\} .
$$

In $[7$, p. 122], it was proved that

$$
\mathcal{H} \backslash\{0\} \subset \mathcal{P}=\mathcal{C}^{*}[(0, \infty)]
$$

and it was told that this is a theorem of F. Hirsch with due reference. This result says that if $f>0$ and $f \in \mathcal{H}$, then $\frac{1}{f}$ is the Laplace transform of a potential kernel, hence the Laplace transform of an infinitely divisible measure and $f$ is a Bernstein function, i.e., a positive function whose derivative is completely monotonic. In [7, p. 127] it is proved that $\mathcal{S} \subset \mathcal{H}$.

From Bernstein's Theorem mentioned above it also follows that completely monotonic functions on $(0, \infty)$ are always strictly completely monotonic unless 
they are constant, see [12], [33, p. 82] and [37, p. 11]. Also it follows that a logarithmically completely monotonic function on $(0, \infty)$ is strictly so unless it is of the form $c \exp (-\alpha x)$ for $c>0$ and $\alpha \geq 0$, so there is no need to discuss the sharpening with "strictly" in general. If its representing measure of a function $f$ as a Stieltjes transform is concentrated on $[a, \infty)$ with $a>0$, then $f \in \mathcal{L}[(-a, \infty)]$.

The classical Euler gamma function is usually defined for $x>0$ by

$$
\Gamma(x)=\int_{0}^{\infty} t^{x-1} e^{-t} \mathrm{~d} t .
$$

The logarithmic derivative of the gamma function

$$
\psi(x)=\frac{\Gamma^{\prime}(x)}{\Gamma(x)}
$$

is called the psi or digamma function and $\psi^{(n)}(x)$ for $n \in \mathbb{N}$ the polygamma functions.

It is well-known that the gamma function is a very important classical special function and has many applications, see [1, 9, 13, 20]. One of the reasons why the gamma function is still interesting, although nearly three centuries have elapsed after its first appearance, is that it has many applications to various areas of mathematics ranging from probability theory to number theory and function theory.

Completely monotonic functions and logarithmically completely monotonic functions have applications in many branches. For example, they play a role in complex analysis, number theory, potential theory, probability theory [9], physics [20], numerical and asymptotic analysis, integral transforms [41], and combinatorics. Some related references are listed in $[2,3,4,5,6,15,23,41]$. In recent years, inequalities and (logarithmically) completely monotonic functions involving the gamma, psi, or polygamma functions are established by some mathematicians (see $[2,3,4,11,15,17,21,31]$ and related references therein).

In this paper, using Leibniz's Identity, the discrete and integral representations of polygamma functions and other analytic techniques, some functions such as

$$
\frac{1}{[\Gamma(x+1)]^{1 / x}}, \quad \frac{[\Gamma(x+1)]^{1 / x}}{x^{\alpha}}, \quad \frac{[\Gamma(x+1)]^{1 / x}}{(x+1)^{\alpha}} \text { and } \quad \frac{[\Gamma(x+\alpha+1)]^{1 /(x+\alpha)}}{[\Gamma(x+1)]^{1 / x}}
$$

with $x \in(-1, \infty)$ or $x \in(0, \infty)$ for $\alpha \in \mathbb{R}$ are showed to be logarithmically completely monotonic. Moreover, some basic properties of the logarithmically completely monotonic functions are established.

Our main results are as follows.

Theorem 1. On the interval $(-1, \infty)$, the reciprocal of the function $[\Gamma(x+$ $1)]^{1 / x}$ is logarithmically completely monotonic, that is,

$$
\frac{1}{[\Gamma(x+1)]^{1 / x}} \in \mathcal{L}[(-1, \infty)]
$$


Theorem 2. Let $\alpha \in \mathbb{R}$. Then

$$
\frac{[\Gamma(x+1)]^{1 / x}}{(x+1)^{\alpha}} \in \mathcal{L}[(-1, \infty)]
$$

if and only if $\alpha \geq 1$.

Let

$$
\tau(s, t)=\frac{1}{s}\left[t-(t+s+1)\left(\frac{t}{t+1}\right)^{s+1}\right]
$$

for $(s, t) \in(0, \infty) \times(0, \infty)$ and $\tau_{0}=\tau\left(s_{0}, t_{0}\right)>0$ be the maximum of $\tau(s, t)$ on the set $\mathbb{N} \times(0, \infty)$. Then for any given real number $\alpha$ satisfying $\alpha \leq \frac{1}{1+\tau_{0}}<1$,

$$
\frac{(x+1)^{\alpha}}{[\Gamma(x+1)]^{1 / x}} \in \mathcal{L}[(-1, \infty)] .
$$

Theorem 3. Let $\alpha \in \mathbb{R}$. Then

$$
\frac{x^{\alpha}}{[\Gamma(x+1)]^{1 / x}} \in \mathcal{L}[(0, \infty)]
$$

if and only if $\alpha \leq 0$. For $\alpha \geq 1$ such that $x^{\alpha}$ is real on $(-1,0)$,

$$
\frac{x^{\alpha}}{[\Gamma(x+1)]^{1 / x}} \in \mathcal{L}[(-1,0)] .
$$

Theorem 4. Let $\alpha \in \mathbb{R}$. Then

$$
\frac{[\Gamma(x+1)]^{1 / x}}{x^{\alpha}} \in \mathcal{L}[(0, \infty)]
$$

if and only if $\alpha \geq 1$. For $\alpha \leq 0$ such that $x^{\alpha}$ is real on $(-1,0)$,

$$
\frac{[\Gamma(x+1)]^{1 / x}}{x^{\alpha}} \in \mathcal{L}[(-1,0)] .
$$

Theorem 5. Logarithmically completely monotonic functions have the following basic properties:

(1) Let $f(x) \in \mathcal{L}[I]$. Then $\frac{f(x)}{f(x+\alpha)} \in \mathcal{L}[J]$ if and only if $\alpha>0$, where $J=I \cap\{x+\alpha \in I\}$. Consequently,

$$
\frac{[\Gamma(x+\alpha+1)]^{1 /(x+\alpha)}}{[\Gamma(x+1)]^{1 / x}} \in \mathcal{L}[(-1, \infty)]
$$

if and only if $\alpha>0$.

(2) Let $h^{\prime}(x) \in \mathcal{C}[I]$ and $f(x) \in \mathcal{L}[h(I)]$. Then $f \circ h(x)=f(h(x)) \in \mathcal{L}[I]$.

In Section 2, we are about to give proofs of these theorems. In Section 3, some remarks are given. In Section 4, some new results are deduced, and some known results are recovered, as applications of the above theorems. 


\section{Proofs of theorems}

It is well-known (see $[1,13,39,40]$ and $[20$, p. 16]) that the polygamma functions $\psi^{(k)}(x)$ can be expressed for $x>0$ and $k \in \mathbb{N}$ as

$$
\psi^{(k)}(x)=(-1)^{k+1} k ! \sum_{i=0}^{\infty} \frac{1}{(x+i)^{k+1}}
$$

or

$$
\psi^{(k)}(x)=(-1)^{k+1} \int_{0}^{\infty} \frac{t^{k} e^{-x t}}{1-e^{-t}} \mathrm{~d} t .
$$

The first proof of Theorem 1. Let

$$
g(x)= \begin{cases}\frac{\ln \Gamma(x+1)}{x}, & x \neq 0 \\ -\gamma, & x=0\end{cases}
$$

for $x \in(-1, \infty)$, where $\gamma=0.57721566 \cdots$ is the Euler-Mascheroni constant. By direct calculation and using Leibniz's Identity, we obtain for $n \in \mathbb{N}$,

$$
g^{(n)}(x)= \begin{cases}\frac{1}{x^{n+1}} \sum_{k=0}^{n} \frac{(-1)^{n-k} n ! x^{k} \psi^{(k-1)}(x+1)}{k !} \triangleq \frac{h_{n}(x)}{x^{n+1}}, & x \neq 0, \\ \frac{\psi^{(n)}(1)}{n+1}, & x=0\end{cases}
$$

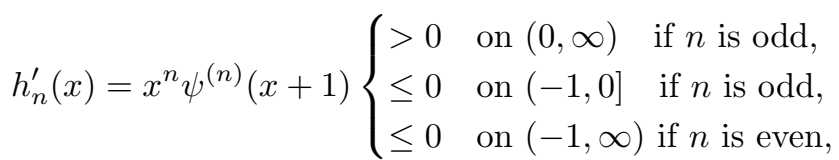

where

$$
\psi^{(-1)}(x+1)=\ln \Gamma(x+1) \quad \text { and } \quad \psi^{(0)}(x+1)=\psi(x+1) .
$$

Hence, if $n$ is odd, the function $h_{n}(x)$ increases on $(0, \infty)$ and decreases on $(-1,0)$, if $n$ is even, it decreases on $(-1, \infty)$. Since $h_{n}(0)=0$, it is easy to see that $h_{n}(x) \geq 0$ on $(-1, \infty)$ if $n$ is odd and that $h_{n}(x) \geq 0$ on $(-1,0)$ and $h_{n}(x) \leq 0$ on $(0, \infty)$ if $n$ is even. Thus, in the interval $(-1, \infty)$, the function $g^{(n)}(x) \geq 0$ if $n$ is odd and $g^{(n)}(x) \leq 0$ if $n$ is even. Since

$$
\lim _{x \rightarrow \infty} \frac{\psi^{(k)}(x+1)}{x^{n-k}}=0
$$

for $-1 \leq k \leq n-1$, it follows that $\lim _{x \rightarrow \infty} g^{(n)}(x)=0$. Consequently,

$$
(-1)^{n+1} g^{(n)}(x)>0
$$

on $(-1, \infty)$ for $n \in \mathbb{N}$. This implies that

$$
(-1)^{k}\left\{\ln [\Gamma(x+1)]^{1 / x}\right\}^{(k)}<0
$$

on $(-1, \infty)$ for $k \in \mathbb{N}$ and the function $\frac{1}{[\Gamma(x+1)]^{1 / x}}$ is logarithmically completely monotonic on $(-1, \infty)$. 
The second proof of Theorem 1. It is not difficult to see that

(26) $g(x)=\frac{\ln \Gamma(x+1)-\ln \Gamma(1)}{x}=\frac{1}{x} \int_{0}^{x} \psi(t+1) \mathrm{d} t=\int_{0}^{1} \psi(x s+1) \mathrm{d} s$

and

$$
g^{(n)}(x)=\int_{0}^{1} s^{n} \psi^{(n)}(x s+1) \mathrm{d} s
$$

Thus, the required result follows from using formula (21) or (22) in (27).

Proof of Theorem 2. Let

$$
\nu_{\alpha}(x)= \begin{cases}\frac{[\Gamma(x+1)]^{1 / x}}{(x+1)^{\alpha}}, & x \neq 0 \\ e^{-\gamma}, & x=0\end{cases}
$$

for $x \in(-1, \infty)$. Then for $n \in \mathbb{N}$, by using $(21)$,

(30) $\quad\left[\ln \nu_{\alpha}(x)\right]^{(n)}=\frac{1}{x^{n+1}}\left[h_{n}(x)+\frac{(-1)^{n}(n-1) ! \alpha x^{n+1}}{(x+1)^{n}}\right] \triangleq \frac{\mu_{\alpha, n}(x)}{x^{n+1}}$,

$$
\begin{aligned}
\mu_{\alpha, n}^{\prime}(x)= & x^{n} \psi^{(n)}(x+1)+\frac{(-1)^{n}(n-1) ! \alpha x^{n}(x+n+1)}{(x+1)^{n+1}} \\
= & x^{n}\left[\psi^{(n)}(x+1)+\frac{(-1)^{n}(n-1) ! \alpha}{(x+1)^{n}}+\frac{(-1)^{n} n ! \alpha}{(x+1)^{n+1}}\right] \\
= & x^{n}\left\{(-1)^{n+1} n ! \sum_{i=1}^{\infty} \frac{1}{(x+i)^{n+1}}\right. \\
& +(-1)^{n}(n-1) ! \alpha \sum_{i=1}^{\infty}\left[\frac{1}{(x+i)^{n}}-\frac{1}{(x+i+1)^{n}}\right] \\
& +(-1)^{n} n ! \alpha \sum_{i=1}^{\infty}\left[\frac{1}{\left.\left.(x+i)^{n+1}-\frac{1}{(x+i+1)^{n+1}}\right]\right\}}\right. \\
= & (-1)^{n}(n-1) ! x^{n} \sum_{i=1}^{\infty}\left[\frac{\alpha}{(x+i)^{n}}-\frac{\alpha}{(x+i+1)^{n}}\right. \\
& \left.-\frac{n \alpha}{(x+i+1)^{n+1}}+\frac{n(\alpha-1)}{(x+i)^{n+1}}\right] \\
= & (n-1) !(-x)^{n} \sum_{i=1}^{\infty} \frac{[\alpha y+n(\alpha-1)](y+1)^{n+1}-\alpha(y+n+1) y^{n+1}}{y^{n+1}(y+1)^{n+1}} \\
& (n y+n)
\end{aligned}
$$




$$
\begin{aligned}
& =(n-1) !(-x)^{n} \sum_{i=1}^{\infty} \frac{\alpha\left[(y+n)(y+1)^{n+1}-(y+n+1) y^{n+1}\right]-n(y+1)^{n+1}}{y^{n+1}(y+1)^{n+1}} \\
& =n !(-x)^{n} \sum_{i=1}^{\infty} \frac{1}{y^{n+1}}\left\{\alpha\left[1+\frac{1}{n}\left\langle y-(y+n+1)\left(\frac{y}{y+1}\right)^{n+1}\right\rangle\right]-1\right\},
\end{aligned}
$$

where $y=x+i>0$.

In [10, p. 28], [18, p. 154] and [19], Bernoulli's inequality states that if $x \geq-1$ and $x \neq 0$ and if $\alpha>1$ or if $\alpha<0$, then

$$
(1+x)^{\alpha}>1+\alpha x .
$$

This means that

which is equivalent to

$$
1+\frac{s+1}{t}<\left(1+\frac{1}{t}\right)^{s+1}
$$

$$
t-(t+s+1)\left(\frac{t}{t+1}\right)^{s+1}>0
$$

for $s>0$ and $t>0$, then the function $\tau(s, t)$ defined by (14) is positive for $(s, t) \in(0, \infty) \times(0, \infty)$.

Since $\tau(s, t)>0$, it is deduced that

$$
[\alpha y+n(\alpha-1)](y+1)^{n+1}-\alpha(y+n+1) y^{n+1}>0
$$

for $y=x+i>0$ and $n \in \mathbb{N}$ if $\alpha \geq 1$. This means that for $\alpha \geq 1$,

$$
\mu_{\alpha, n}^{\prime}(x) \begin{cases}>0 & \text { on }(-1,0) \cup(0, \infty) \text { if } n \text { is even, } \\ >0 & \text { on }(-1,0) \text { if } n \text { is odd, } \\ <0 & \text { on }(0, \infty) \text { if } n \text { is odd, }\end{cases}
$$

hence, it is obtained that the function $\mu_{\alpha, n}(x)$ is strictly increasing on $(-1, \infty)$ if $n$ is even and that the function $\mu_{\alpha, n}(x)$ is strictly increasing on $(-1,0)$ and strictly decreasing on $(0, \infty)$ if $n$ is odd. Since $\mu_{\alpha, n}(0)=0$, it follows that $\mu_{\alpha, n}(x) \leq 0$ on $(-1, \infty)$ if $n$ is odd and that $\mu_{\alpha, n}(x) \leq 0$ on $(-1,0)$ and $\mu_{\alpha, n}(x)>0$ on $(0, \infty)$ if $n$ is even. From $\lim _{x \rightarrow \infty}\left[\ln \nu_{\alpha}(x)\right]^{(n)}=0$, it is concluded that $\left[\ln \nu_{\alpha}(x)\right]^{(n)} \geq 0$ on $(-1, \infty)$ if $n$ is even and $\left[\ln \nu_{\alpha}(x)\right]^{(n)} \leq 0$ on $(-1, \infty)$ if $n$ is odd, which is equivalent to $(-1)^{n}\left[\ln \nu_{\alpha}(x)\right]^{(n)}>0$ on $x \in(-1, \infty)$ for $n \in \mathbb{N}$ and $\alpha \geq 1$. Hence, if $\alpha \geq 1$, the function $\frac{[\Gamma(x+1)]^{1 / x}}{(x+1)^{\alpha}}$ is logarithmically completely monotonic on $(-1, \infty)$.

Conversely, if the function $\frac{[\Gamma(x+1)]^{1 / x}}{(x+1)^{\alpha}}$ is logarithmically completely monotonic on $(-1, \infty)$, then $\left[\ln \nu_{\alpha}(x)\right]^{\prime} \leq 0$ which is equivalent to

$$
\begin{aligned}
\alpha & \geq \frac{x+1}{x^{2}}[x \psi(x+1)-\ln \Gamma(x+1)] \\
& =\left(1+\frac{1}{x}\right)\left[\psi(x+1)-\frac{\ln \Gamma(x+1)}{x}\right]
\end{aligned}
$$




$$
\begin{aligned}
& \rightarrow\left(1+\frac{1}{x}\right)\left\{\ln (x+1)-\frac{1}{2(x+1)}-\frac{1}{12(x+1)^{2}}+O\left(\frac{1}{x+1}\right)\right. \\
& \left.-\frac{1}{x}\left[\left(x+\frac{1}{2}\right) \ln (x+1)-x-1+\frac{\ln (2 \pi)}{2}+\frac{1}{12(x+1)}+O\left(\frac{1}{x+1}\right)\right]\right\} \\
& \rightarrow 1
\end{aligned}
$$

as $x \rightarrow \infty$ by using the following formulas (see $[1,20,39,40]$ )

$$
\ln \Gamma(x)=\left(x-\frac{1}{2}\right) \ln x-x+\frac{\ln (2 \pi)}{2}+\frac{1}{12 x}+O\left(\frac{1}{x}\right)
$$

and

$$
\psi(x)=\ln x-\frac{1}{2 x}-\frac{1}{12 x^{2}}+O\left(\frac{1}{x^{2}}\right)
$$

as $x \rightarrow \infty$.

Since $\tau(s, t)>0$ for $(s, t) \in(0, \infty) \times(0, \infty)$, we have $\tau_{0}>0$. When $\alpha \leq$ $\frac{1}{1+\tau_{0}}<1$, it follows from $(31)$ that $\mu_{\alpha, n}^{\prime}(x) \leq 0$ and $\mu_{\alpha, n}(x)$ is decreasing in $(-1, \infty)$ if $n$ an even integer and that $\mu_{\alpha, n}^{\prime}(x) \leq 0$ and $\mu_{\alpha, n}(x)$ is decreasing on $(-1,0)$ and $\mu_{\alpha, n}^{\prime}(x) \geq 0$ and $\mu_{\alpha, n}(x)$ is increasing on $(0, \infty)$ if $n$ an odd integer. Since $\mu_{\alpha, n}(0)=0$ and $\lim _{x \rightarrow \infty}\left[\ln \nu_{\alpha}(x)\right]^{(n)}=0$, we have $\left[\ln \nu_{\alpha}(x)\right]^{(n)}<0$ for $n$ being an even and $\left[\ln \nu_{\alpha}(x)\right]^{(n)}>0$ for $n$ being an odd on $(-1, \infty)$, this implies that $(-1)^{n+1}\left[\ln \nu_{\alpha}(x)\right]^{(n)}>0$ on $(-1, \infty)$ for $n \in \mathbb{N}$. Therefore $\nu_{\alpha}(x)$ is strictly increasing and $(-1)^{n-1}\left\{\left[\ln \nu_{\alpha}(x)\right]^{\prime}\right\}^{(n-1)}>0$ on $(-1, \infty)$ for $n \in \mathbb{N}$. Hence, if $\alpha \leq \frac{1}{1+\tau_{0}}$, then the function $\frac{(x+1)^{\alpha}}{[\Gamma(x+1)]^{1 / x}}$ is logarithmically completely monotonic on $(-1, \infty)$. The proof of Theorem 2 is complete.

Second proof of sufficiency for (13). By [8, p. 522, Theorem 5.1], it is easy to obtain that

$$
h(z)=\frac{\ln \Gamma(z+1)}{z}-\alpha \ln (z+1)=c+\int_{1}^{\infty}\left(\frac{1}{t+z}-\frac{t}{1+t^{2}}\right)[\alpha-M(t)] \mathrm{d} t
$$

with

$$
c=-\gamma+\sum_{k=1}^{\infty}\left(\frac{1}{k}-\arctan \frac{1}{k}\right)
$$

and $M(t)=\frac{k-1}{t}$ for $t \in(k-1, k]$ and $k=2,3, \ldots$ For $\alpha \geq 1$ one has $\alpha \geq M(t)$. Accordingly,

$$
h^{\prime}(t)=-\int_{1}^{\infty} \frac{\alpha-M(t)}{(t+z)^{2}} \mathrm{~d} t<0, \quad z>-1
$$

that is, the function $h$ is decreasing with $-h^{\prime} \in \mathcal{C}[(-1, \infty)]$, which is the sufficient part of (13).

Proof of Theorem 3. If $\alpha \leq 0$, the logarithmically complete monotonicity of the function $\frac{x^{\alpha}}{[\Gamma(x+1)]^{1 / x}}$ on $(0, \infty)$ follows from the similar arguments as in the proofs of Theorem 2 . 
If $\frac{x^{\alpha}}{[\Gamma(x+1)]^{1 / x}}$ on $(0, \infty)$ is logarithmically completely monotonic, then its logarithmic derivative

$$
\frac{\alpha}{x}+\frac{\ln \Gamma(1+x)-x \psi(1+x)}{x^{2}}
$$

is negative on $(0, \infty)$. Since

$$
\lim _{x \rightarrow 0^{+}} \frac{\ln \Gamma(1+x)-x \psi(1+x)}{x^{2}}=-\frac{\pi^{2}}{12}
$$

by L'Hospital's rule and

$$
\lim _{x \rightarrow 0^{+}} \frac{\alpha}{x}= \begin{cases}\infty, & \text { if } \alpha>0, \\ 0, & \text { if } \alpha=0 \\ -\infty, & \text { if } \alpha<0\end{cases}
$$

then it must hold that $\alpha \leq 0$.

The rest proofs of Theorem 3 are similar to the proofs of Theorem 2, so we omit them.

Proof of Theorem 4. This follows from modified arguments of above theorems.

Proof of Theorem 5. Let $\mathcal{F}_{\alpha}(x)=\frac{f(x)}{f(x+\alpha)}$ for $\alpha>0$. Since $f(x)$ is logarithmically completely monotonic, by definition we have $(-1)^{k}[\ln f(x)]^{(k)} \geq 0$ for $k \in \mathbb{N}$, which is equivalent to $[\ln f(x)]^{(2 i)} \geq 0$ and $[\ln f(x)]^{(2 i-1)} \leq 0$ for $i \in \mathbb{N}$, and $[\ln f(x)]^{(2 i)}$ is decreasing and $[\ln f(x)]^{(2 i-1)}$ is increasing. So

$$
\left[\ln \mathcal{F}_{\alpha}(x)\right]^{(2 i)}=[\ln f(x)]^{(2 i)}-[\ln f(x+\alpha)]^{(2 i)} \geq 0
$$

and $\left[\ln \mathcal{F}_{\alpha}(x)\right]^{(2 i-1)} \leq 0$ for $\alpha>0$ and $i \in \mathbb{N}$.

The property (20) follows from Theorem 1 and the monotonicity of $\mathcal{F}_{\alpha}(x)$.

In $[14$, No. 0.430.1] the formula for the $n$-th derivative of a composite function is given by

$$
\frac{\mathrm{d}^{n}}{\mathrm{~d} x^{n}}[f(h(x))]=\sum_{k=1}^{n} \frac{1}{k !} f^{(k)}(h(x)) U_{k}(x),
$$

where

$$
U_{k}(x)=\sum_{i=0}^{k-1}(-1)^{i}\left(\begin{array}{l}
k \\
i
\end{array}\right)[h(x)]^{i} \frac{\mathrm{d}^{n}}{\mathrm{~d} x^{n}}[h(x)]^{k-i} .
$$

From this it is deduced that $-\frac{f^{\prime}(h(x))}{f(h(x))} \in \mathcal{C}[I]$ since $-\frac{f^{\prime}(x)}{f(x)}$ is a completely monotonic function, which is equivalent to $f(x)$ being logarithmically completely monotonic, and $h^{\prime}(x) \in \mathcal{C}[I]$. Therefore, $(-1)^{i}\left[\frac{f^{\prime}(h(x))}{f(h(x))}\right]^{(i)} \leq 0$ on the interval $I$ for nonnegative integer $i$.

Since $h^{\prime}(x) \in \mathcal{C}[I]$, it is obtained that $(-1)^{i} h^{(i+1)}(x) \geq 0$ on the interval $I$ for nonnegative integer $i$. 
Hence, for $k \in \mathbb{N}$,

$$
\begin{aligned}
& (-1)^{k}[\ln f(h(x))]^{(k)}=(-1)^{k}\left[\frac{f^{\prime}(h(x))}{f(h(x))} h^{\prime}(x)\right]^{(k-1)} \\
= & (-1)^{k} \sum_{i=0}^{k-1}\left(\begin{array}{c}
k-1 \\
i
\end{array}\right)\left[\frac{f^{\prime}(h(x))}{f(h(x))}\right]^{(i)} h^{(k-i)}(x) \\
= & \sum_{i=0}^{k-1}\left(\begin{array}{c}
k-1 \\
i
\end{array}\right)\left\{(-1)^{i}\left[\frac{f^{\prime}(h(x))}{f(h(x))}\right]^{(i)}\right\}\left[(-1)^{k-i} h^{(k-i)}(x)\right] \geq 0 .
\end{aligned}
$$

The proof of Theorem 5 is complete.

\section{Remarks}

After proving our theorems, we would like to give several remarks on them.

Remark 1. As said in [6] and done in various papers, the complete monotonicity for special functions has been established by proving the stronger statement that the function is logarithmically completely monotonic or is a Stieltjes transform. In some concrete cases it is often easier to establish that a function is logarithmically completely monotonic or is a Stieltjes transform than to verify directly the complete monotonicity. One of the important values of this paper might be owning to the standard or elementary proofs of some theorems in this paper.

Remark 2. It is remarked that many complete monotonicity results in $[2,3$, $4,11,21]$ and closely-related references therein can be restated indeed as the logarithmically complete monotonicity.

Remark 3. In [17, 22], the following monotonicity results were obtained:

$$
\begin{gathered}
{[\Gamma(1+k)]^{1 / k}<[\Gamma(2+k)]^{1 /(k+1)}, \quad k \in \mathbb{N} ;} \\
{\left[\Gamma\left(1+\frac{1}{x}\right)\right]^{x} \text { decreases with } x>0 .}
\end{gathered}
$$

These are extended and generalized in $[25,34]$ : The function $[\Gamma(r)]^{1 /(r-1)}$ is increasing in $r>0$. Clearly, Theorem 1 generalizes these results and extends them for the range of the argument.

The property (20) implies that the sequences

$$
\frac{\sqrt[k]{k !}}{\sqrt[m+k]{(m+k) !}} \text { and } \frac{[\sqrt[k]{k !}][\sqrt[k+m+n]{(k+m+n) !}]}{[\sqrt[k+m]{(k+m) !}][\sqrt[k+n]{(k+n) !}]}
$$

are increasing with $k \in \mathbb{N}$ for given natural numbers $m$ and $n$.

Remark 4. It is proved in [27] that

$$
\frac{\ln \Gamma(x+1)}{x}-\ln x+1=\ln \frac{[\Gamma(x+1)]^{1 / x}}{x}+1 \in \mathcal{C}[(0, \infty)]
$$


A similar result was found in [38]: The function

$$
1+\frac{\ln \Gamma(x+1)}{x}-\ln (x+1)=\ln \frac{[\Gamma(x+1)]^{1 / x}}{x+1}+1 \in \mathcal{C}[(-1, \infty)] .
$$

These are special cases of Theorems 2 and 4 .

Remark 5. The property (20) generalizes and extends those in [29].

Remark 6. It has been proved in the proof of Theorem 2 that $\tau(s, t)>0$ for $(s, t) \in(0, \infty) \times(0, \infty)$. Now we give an upper bound of the function $\tau(s, t)$ on $(0, \infty) \times(0, \infty)$

Let $s=\mu t$ for $\mu \in(0, \infty)$. Then we have

$$
\tau(\mu t, t)=\frac{1}{\mu}\left[1-\frac{(\mu+1) t+1}{1+t}\left(\frac{t}{1+t}\right)^{\mu t}\right] .
$$

Since the function $\frac{(\mu+1) t+1}{1+t}$ is strictly increasing with $t \in(0, \infty)$ for fixed $\mu \in$ $(0, \infty)$, it follows that

$$
1<\frac{(\mu+1) t+1}{1+t}<\mu+1 .
$$

Since the function $\left(1+\frac{1}{t}\right)^{t}$ is strictly increasing, we see that

$$
\left(\frac{t}{1+t}\right)^{\mu t}=\left[\frac{1}{(1+1 / t)^{t}}\right]^{\mu}
$$

is strictly decreasing with $t \in(0, \infty)$ for fixed $\mu \in(0, \infty)$, therefore

$$
\frac{1}{e^{\mu}}<\left(\frac{t}{1+t}\right)^{\mu t}<1
$$

Combining (40), (41) and (43) produces

$$
\tau(\mu t, t)<\frac{1}{\mu}\left(1-\frac{1}{e^{\mu}}\right)<\lim _{\mu \rightarrow 0}\left[\frac{1}{\mu}\left(1-\frac{1}{e^{\mu}}\right)\right]=1 .
$$

Since $\mu \in(0, \infty)$ and $t \in(0, \infty)$ are arbitrary, so we have

$$
\tau(s, t)<1
$$

for $(s, t) \in(0, \infty) \times(0, \infty)$.

Recently, the inequality (45) was improved in $[26,35]$.

\section{Applications}

In what follows, as applications of our main results, we would like to deduce some consequences of our theorems stated in Section 1.

The first application of our theorems is the following proposition. 
Proposition 1. For $\alpha \geq 1$ and $\beta>0$, the function

$$
\frac{[\Gamma(x+1)]^{1 / x}}{[\Gamma(x+1+\beta)]^{1 /(x+\beta)}}\left(1+\frac{\beta}{x+1}\right)^{\alpha} \in \mathcal{L}[(-1, \infty)] .
$$

For $\beta>0$ and any given real number $\alpha$ satisfying $\alpha \leq \frac{1}{1+\tau_{0}}<1$, the reciprocal of the function defined in (46) belongs to $\mathcal{L}[(-1, \infty)]$.

For $\alpha \geq 1$ and $\beta>0$, the function

$$
\frac{[\Gamma(x+1)]^{1 / x}}{[\Gamma(x+1+\beta)]^{1 /(x+\beta)}}\left(1+\frac{\beta}{x}\right)^{\alpha} \in \mathcal{L}[(0, \infty)] .
$$

For $\alpha \leq 0$ and $\beta>0$, the reciprocal of the function defined in (47) belongs to $\mathcal{L}[(0, \infty)]$.

Proof. These follow from combining Theorem 5 with Theorem 1 to Theorem 4 .

Remark 7. The results in Proposition 1 generalize and extend those of [29].

Define

$$
Q_{a, b}(x)=\frac{[\Gamma(x+a+1)]^{1 /(x+a)}}{[\Gamma(x+b+1)]^{1 /(x+b)}}
$$

for nonnegative real numbers $a$ and $b$. J. Sándor [36] established that $Q_{1,0}$ is decreasing on $(1, \infty)$. In [4] Alzer and Berg proved that $\left[Q_{a, b}(x)\right]^{c}$ is completely monotonic with $x \in(0, \infty)$ if and only if $a \geq b$ for $c>0$. The following proposition extends the ranges of variables $a, b$ and $x$ in [4] and can be regarded as a generalization of Proposition 1 above.

Proposition 2. Let $a, b \in \mathbb{R}$ and $c>0$. Then $\left[Q_{a, b}(x)\right]^{c} \in \mathcal{L}[(-(1+b), \infty)]$ if and only if $a>b$.

Proof. From Theorem 1, it is clear that

$$
\frac{1}{[\Gamma(x+a+1)]^{1 /(x+a)}} \in \mathcal{L}[(-(1+a), \infty)]
$$

for $a \in \mathbb{R}$. From Theorem 5 it follows that the function $Q_{a, b}(x)$ is logarithmically completely monotonic on $(-(1+a), \infty) \cap(-(1+b), \infty)=(-(1+b), \infty)$ for $a>b$. So does the function $\left[Q_{a, b}(x)\right]^{c}$ for $c>0$.

If $\left[Q_{a, b}(x)\right]^{c}$ is logarithmically completely monotonic for $c>0$, then the derivative $\left\{\left[\ln Q_{a, b}(x)\right]^{c}\right\}^{\prime}=c\left[g^{\prime}(x+a)-g^{\prime}(x+b)\right]<0$, where $g(x)$ is defined by $(23)$ and $g^{\prime}(x)$ is strictly decreasing on $(-1, \infty)$, since $g^{\prime \prime}(x)=\int_{0}^{1} t^{2} \psi^{\prime \prime}(x t+$ 1) $\mathrm{d} t<0$ from (27) and (21). Therefore, there must be $a>b$.

As consequences of the third conclusion in Theorem 5, we have: 
Proposition 3. Let $f$ be a logarithmically completely monotonic function and $g$ a completely monotonic function. Then the function

$$
f\left(a+b \int_{\alpha}^{x} g(t) \mathrm{d} t\right)
$$

is logarithmically completely monotonic on an interval I if it is defined on I, where $b$ is positive and $\alpha \in I$.

In particular, if $f$ is logarithmically completely monotonic, then the following functions are also logarithmically completely monotonic:

(49) $f\left(a x^{\alpha}+b\right)$, where $a$ is nonnegative numbers and $0 \leq \alpha \leq 1$,

$$
\begin{gathered}
f(a+b \ln (1+x)), \quad \text { where } b \text { is nonnegative, } \\
f\left(1-e^{-x}\right), \\
f(\arctan \sqrt{x}) .
\end{gathered}
$$

If $f(x)$ is completely monotonic on an interval $I$, then the function $[A-$ $f(x)]^{-\mu}$ is logarithmically completely monotonic on $I$, where $A>f(x)$ for $x \in I$ and $\mu \geq 0$.

Proof. These are direct consequences of Theorem 5.

Remark 8 . The following functions are also logarithmically completely monotonic:

$$
\begin{gathered}
\exp \left(-a x^{\alpha}\right), \quad \text { where } a \geq 0 \text { and } 0 \leq \alpha \leq 1, \\
{[a+b \ln (1+x)]^{-\mu}, \quad \text { where } a \geq 0, b \geq 0 \text { and } \mu>0,} \\
\left(a-b e^{-x}\right)^{-\mu}, \quad \text { where } a \geq b>0 \text { and } \mu \geq 0 .
\end{gathered}
$$

Remark 9. Finally, we pose an open problem: Let $\tau_{0}=\tau\left(s_{0}, t_{0}\right)$ be the maximum value of $\tau(s, t)$ defined by (14) on the set $\mathbb{N} \times(0, \infty)$. Then

$$
\frac{(x+1)^{\alpha}}{[\Gamma(x+1)]^{1 / x}} \in \mathcal{L}[(-1, \infty)]
$$

if and only if $\alpha \leq \frac{1}{1+\tau_{0}}<1$.

Remark 10. This paper is a slightly revised version of the preprints $[28,30]$.

Acknowledgments. The authors appreciate the anonymous referees for their helpful comments on and valuable suggestions to the original version of this manuscript.

\section{References}

[1] M. Abramowitz and I. A. Stegun, Handbook of Mathematical Functions with Formulas, Graphs, and Mathematical Tables, Third printing, with corrections. National Bureau of Standards Applied Mathematics Series, 55 Superintendent of Documents, U.S. Government Printing Office, Washington, D.C. 1965.

[2] H. Alzer, On some inequalities for the gamma and psi functions, Math. Comp. 66 (1997), no. 217, 373-389. 
[3] H. Alzer and C. Berg, Some classes of completely monotonic functions, Ann. Acad. Sci. Fenn. Math. 27 (2002), no. 2, 445-460.

[4] Some classes of completely monotonic functions. II, Ramanujan J. 11 (2006), no. $2,225-248$

[5] R. D. Atanassov and U. V. Tsoukrovski, Some properties of a class of logarithmically completely monotonic functions, C. R. Acad. Bulgare Sci. 41 (1988), no. 2, 21-23.

[6] C. Berg, Integral representation of some functions related to the gamma function, Mediterr. J. Math. 1 (2004), no. 4, 433-439.

[7] C. Berg and G. Forst, Potential Theory on Locally Compact Abelian Groups, SpringerVerlag, New York-Heidelberg, 1975.

[8] C. Berg and H. L. Pedersen, Pick functions related to the gamma function, Rocky Mountain J. Math. 32 (2002), no. 2, 507-525.

[9] S. Bochner, Harmonic Analysis and the Theory of Probability, University of California Press, Berkeley and Los Angeles, 1955.

[10] P. S. Bullen, A Dictionary of Inequalities, Pitman Monographs and Surveys in Pure and Applied Mathematics, 97. Longman, Harlow, 1998.

[11] W. E. Clark and M. E. H. Ismail, Inequalities involving gamma and psi functions, Anal. Appl. (Singap.) 1 (2003), no. 1, 129-140.

[12] M. J. Dubourdieu, Sur un théorème de M. S. Bernstein relatif à la transformation de Laplace-Stieltjes, Compositio Math. 7 (1939), 96-111.

[13] A. Erdélyi, W. Magnus, F. Oberhettinger, and F. Tricomi, Higher Transcendental Functions. Vols. I, II, McGraw-Hill Book Company, Inc., New York-Toronto-London, 1953.

[14] I. S. Gradshtein and I. M. Ryzhik, Tables of Integrals, Sums, Series and Products, 5th Edition, Academic Press, 1994.

[15] A. Z. Grinshpan and M. E. H. Ismail, Completely monotonic functions involving the gamma and q-gamma functions, Proc. Amer. Math. Soc. 134 (2006), no. 4, 1153-1160.

[16] R. A. Horn, On infinitely divisible matrices, kernels, and functions, Z. Wahrscheinlichkeitstheorie und Verw. Gebiete 8 (1967), 219-230.

[17] D. Kershaw and A. Laforgia, Monotonicity results for the gamma function, Atti Accad. Sci. Torino Cl. Sci. Fis. Mat. Natur. 119 (1985), no. 3-4, 127-133.

[18] J.-Ch. Kuang, Applied Inequalities, Second edition. With a preface by Shan Zhen Lu. Hunan Jiaoyu Chubanshe, Changsha, 1993.

[19] _ Applied Inequalities, Third edition. Shandong Science and Technology Press, Ji'nan City, Shandong Province, China, 2004

[20] W. Magnus, F. Oberhettinger, and R. P. Soni, Formulas and Theorems for the Special Functions of Mathematical Physics, Springer, Berlin, 1966.

[21] K. S. Miller and S. G. Samko, Completely monotonic functions, Integral Transform. Spec. Funct. 12 (2001), no. 4, 389-402.

[22] H. Minc and L. Sathre, Some inequalities involving $(r !)^{1 / r}$, Proc. Edinburgh Math. Soc. (2) $14(1964 / 1965), 41-46$.

[23] D. S. Mitrinović, J. E. Pečarić, and A. M. Fink, Classical and New Inequalities in Analysis, Kluwer Academic Publishers Group, Dordrecht, 1993

[24] J. Pečarić, F. Proschan, and Y. L. Tong, Convex Functions, Partial Orderings, and Statistical Applications, Mathematics in Science and Engineering, 187. Academic Press, Inc., Boston, MA, 1992.

[25] F. Qi, Monotonicity results and inequalities for the gamma and incomplete gamma functions, Math. Inequal. Appl. 5 (2002), no. 1, 61-67.

[26] F. Qi, J. Cao, D.-W. Niu, and N. Ujevic, An upper bound of a function with two independent variables, Appl. Math. E-Notes 6 (2006), 148-152.

[27] F. Qi and Ch.-P. Chen, A complete monotonicity property of the gamma function, J. Math. Anal. Appl. 296 (2004), no. 2, 603-607. 
[28] F. Qi and B.-N. Guo, Complete monotonicities of functions involving the gamma and digamma functions, RGMIA Res. Rep. Coll. 7 (2004), no. 1, Art. 8, 63-72; Available online at http://rgmia.org/v7n1.php.

[29] _ Monotonicity and convexity of ratio between gamma functions to different powers, J. Indones. Math. Soc. 11 (2005), no. 1, 39-49.

[30] _ Some logarithmically completely monotonic functions related to the gamma function, Available online at http://arxiv.org/abs/0903.5123.

[31] F. Qi, B.-N. Guo, and Ch.-P. Chen, The best bounds in Gautschi-Kershaw inequalities, Math. Inequal. Appl. 9 (2006), no. 3, 427-436.

[32] _ Some completely monotonic functions involving the gamma and polygamma functions, RGMIA Res. Rep. Coll. 7 (2004), no. 1, Art. 5, 31-36; Available online at http://rgmia.org/v7n1.php.

[33] - Some completely monotonic functions involving the gamma and polygamma functions, J. Aust. Math. Soc. 80 (2006), no. 1, 81-88.

[34] F. Qi and S. Guo, On a new generalization of Martins' inequality, J. Math. Inequal. 1 (2007), no. 4, 503-514.

[35] F. Qi, D.-W. Niu, and J. Cao, An infimum and an upper bound of a function with two independent variables, Octogon Math. Mag. 14 (2006), no. 1, 248-250.

[36] J. Sándor, Sur la fonction gamma, Publ. C. R. M. P. Neuchâtel, Série I 21 (1989), 4-7.

[37] H. van Haeringen, Completely Monotonic and Related Functions, Report 93-108, Faculty of Technical Mathematics and Informatics, Delft University of Technology, Delft, The Netherlands, 1993.

[38] H. Vogt and J. Voigt, A monotonicity property of the $\Gamma$-function, J. Inequal. Pure Appl. Math. 3 (2002), no. 5, Article 73, 3 pp.; Available online at http://jipam.vu.edu.au/ article.php?sid=225.

[39] Zh.-X. Wang and D.-R. Guo, Special Functions, Translated from the Chinese by Guo and X. J. Xia. World Scientific Publishing Co., Inc., Teaneck, NJ, 1989.

[40] _ A Panorama of Special Functions, The Series of Advanced Physics of Peking University, Peking University Press, Beijing, China, 2000.

[41] D. V. Widder, The Laplace Transform, Princeton University Press, Princeton, 1941.

FENG QI

SCHOOL OF MATHEMATICS AND INFORMATICS

Henan Polytechnic University

JiAOZUO City

Henan Province, 454010, P. R. China

E-mail address: qifeng618@gmail.com, qifeng618@hotmail.com, qifeng618@qq.com

BAI-Ni GuO

SCHOOL OF MATHEMATICS AND INFORMATICS

Henan Polytechnic University

JiaOzuo City

Henan Province, 454010, P. R. China

E-mail address: bai.ni.guo@gmail.com, bai.ni.guo@hotmail.com 